\title{
On the convergence of piecewise linear strategic interaction dynamics on networks
}

\author{
Bahman Gharesifard
}

\author{
Behrouz Touri
}

\author{
Tamer Başar
}

Jeff Shamma

\begin{abstract}
We prove that the piecewise linear best-response dynamical systems of strategic interactions are asymptotically convergent to their set of equilibria on any weighted undirected graph. We study various features of these dynamical systems, including the uniqueness and abundance properties of the set of equilibria and the emergence of unstable equilibria. We also introduce the novel notions of social equivalence and social dominance on directed graphs, and demonstrate some of their interesting implications, including their correspondence to consensus and chromatic number of partite graphs. Examples illustrate our results.
\end{abstract}

\section{INTRODUCTION}

Various engineering and economic systems consist of collections of smaller subsystems, interconnected over a network. Examples include oscillator synchronization [2], distributed robotic networks [3], and cascades of information and opinions in social networks; see [4] and references therein. The diverse nature of interconnections in these systems makes it difficult to classify them in any useful manner. One, perhaps naive, approach is to prescribe the behavior of each individual by using a payoff function which is dependent on the actions taken by neighboring subsystems. Each subsystem, which we often refer to as an agent, then aims to maximize its payoff function. In most engineering scenarios, there is a designer whose objective is to assign appropriate such functions to ensure certain performances, while still allowing for execution in a decentralized manner. In economic scenarios, endowing individuals with payoff functions is a rather natural starting point, which calls for a game-theoretic framework for analyzing their decision making processes.

The global behavior of the systems alluded to above are often prescribed as the asymptotics of some dynamical systems whose evolutions capture the responses of individuals to actions of others. These dynamical systems are coupled through local interconnections. Clearly, the topology of interconnections plays a central role in the study of these limiting behaviors. The dynamical systems resulting from the gametheoretic modeling of interactions on networks have a variety of applications in engineering systems, including distributed optimization [5], resource allocation [6], and analysis of competition between firms [7].

This paper is concerned with characterizing the limiting behavior of an important sub-class of such systems, namely the

Bahman Gharesifard is with the Queen's University, Canada. Behrouz Touri is with the University of Colorado Boulder, USA. Tamer Başar is with the University of Illinois at Urbana-Champaign, USA. Jeff Shamma is with the King Abdullah University of Science and Technology (KAUST), Saudi Arabia, and Georgia Institute of Technology, USA. Email: bahman@mast.queensu.ca, behrouz.touriecolorado.edu, basarleillinois.edu, and shammalgatech.edu. An incomplete version of a portion of this paper was presented as [1] in the 2013 IEEE Control and Decision Conference. piecewise linear best-response dynamical systems of strategic interactions. These dynamics describe scenarios in which the payoff of each agent is positively enhanced by the investments of neighboring individuals and have recently been studied (see [8]). Each agent aims at providing a unit of resource and invests only if this unit is not provided, collectively by itself and its neighboring agents. The rate of contributions of each agent is inversely proportional to the investments of others. In this sense, the model is a competitive threshold model [9] or exhibits strategic substitutability [10]. The recent survey [11] summarizes the importance of these models and their applications, including Cournot competition [8] and private provision of public goods and investment in security [12], see also [13], [14]. The recent work [15] studies the limiting behavior of piecewise linear best-response dynamical systems for some scenarios with asymmetric interactions.

A distinct aspect of the current work is that it is concerned with the limiting properties of this class in spite of the heterogenous character; in particular, unlike the results known in the literature, we study scenarios of sparse networks where strong dependencies on neighboring actions result in complex phenomena, including the abundance of equilibria and the emergence of unstable equilibria in stable submanifolds. These complex features are common with other similar cascades [7]. It is worth mentioning that the technical part of this work is related to the literature on stability analysis of piecewise linear systems [16], [17], [18], notions of potential games [19], cooperative and noncooperative dynamical systems [20], [21], and best-response dynamics [22], [23], [24].

Our contributions in this paper are threefold. First, we prove that the piecewise linear best-response dynamical systems of strategic interactions are asymptotically convergent when the network topology is undirected, thus extending the class of systems for which these dynamics are known to converge. Our proof relies on the asymptotic stability properties of continuous-time best-response dynamical systems for potential games with component-wise concave potential functions. Next, we demonstrate interesting features of the set of equilibria of these dynamical systems, including the uniqueness and abundance properties of the set of equilibria and the possibility of emergence of unstable equilibria in stable submanifolds. We discuss applications of these dynamical systems in stability analysis of On-Off Systems. We also introduce the novel notions of social equivalence and social dominance, and show some of their interesting implications, including their connections to consensus. Several examples included in the paper illustrate the results. 


\section{MathematicAl PRELIMINARIES}

We start with some notational conventions. Let $\mathbb{R}, \mathbb{C}, \mathbb{R}_{\geq 0}$, $\mathbb{Z}$, and $\mathbb{Z}_{\geq 1}$ denote the sets of real, complex, nonnegative real, integer, and positive integer numbers, respectively. The set of eigenvalues of a matrix $A \in \mathbb{R}^{n \times n}, n \in \mathbb{Z}_{\geq 1}$, is denoted by $\operatorname{spec}(A) \subset \mathbb{C}$. When $A$ is symmetric, we denote by $\lambda_{\min }(A)$ its minimum eigenvalue. We denote by $\phi: X \rightrightarrows Y$ the setvalued mapping $\phi$ from the set $X$ to the set $Y$.

A directed graph, or simply digraph, is a pair $\mathcal{G}=(V, E)$, where $V$ is a finite set called the vertex set and $E \subseteq V \times V$ is the edge set. A weighted digraph is a triplet $\mathcal{G}=(V, E, A)$, where $(V, E)$ is a digraph and $A \in \mathbb{R}_{>0}^{n \times n}$ is the adjacency matrix. We denote the entries of $A$ by $a_{i j}, i, j \in\{1, \ldots, n\}$, with entry $a_{i j}>0$ if and only if $\left(v_{i}, v_{j}\right) \in E$, and $a_{i j}=0$ otherwise. If a matrix $A$ satisfies this property, we say that $A$ is a weight assignment of the digraph $\mathcal{G}=(V, E)$. The out- and indegrees of $v_{i} \in V$ are, respectively, $d_{\mathrm{out}}^{\mathrm{w}}\left(v_{i}\right)=\sum_{j=1}^{n} a_{i j}$ and $d_{\mathrm{in}}^{\mathrm{w}}\left(v_{i}\right)=\sum_{j=1}^{n} a_{j i}$. Unless mentioned otherwise, we further assume that $a_{i j}=1$ when $\left(v_{i}, v_{j}\right) \in E$ and that $a_{i i}=0$ for all $i \in\{1, \ldots, n\}$. We also let $\mathcal{N}_{i}^{+}=\left\{u \in V \mid\left(v_{i}, u\right) \in E\right\}$ and refer to it as the set of out-neighbors of $v_{i}$.

In the paper we work primarily with the class of undirected graphs, or simply graphs, where the edge set $E$ consists of unordered pairs of vertices. The degree of a vertex $v \in V$, denoted $d(v)$, is the number of neighbors of $v$. For a weighted graph, the weighted degree of $v_{i}, i \in\{1, \ldots, n\}$, is $d^{\mathrm{w}}\left(v_{i}\right)=$ $\sum_{j=1}^{n} a_{i j}=\sum_{j=1}^{n} a_{j i}$. For an undirected graph, we simply refer to the the set $\mathcal{N}_{i}$ as the set of neighbors of $v_{i}$.

Finally, we recall some background on continuous-time dynamical systems following [25]. Consider a system on $X \subset \mathbb{R}^{d}$ given by

$$
\dot{x}(t)=\Psi(x(t)),
$$

where $t \in \mathbb{R}_{\geq 0}$ and $\Psi: X \subset \mathbb{R}^{d} \rightarrow \mathbb{R}^{d}$ is continuous. A solution to this dynamical system is a continuously differentiable curve $x:[0, T] \rightarrow X$ which satisfies (1). The set of equilibria of (1) is denoted by $\mathrm{Eq}(\Psi)=\{x \in X \mid \Psi(x)=0\}$.

\section{Problem Statement}

Consider a weighted digraph $\mathcal{G}=(V, E, A)$ with $n \in \mathbb{Z}_{\geq 1}$ vertices. Throughout the paper, we study dynamical systems on $\mathcal{G}$, where the evolution of the state of agent $v_{i} \in V$ is

$$
\dot{x}_{i}=\max \left\{1-\sum_{j=1}^{n} a_{i j} x_{j}, 0\right\}-x_{i}, \quad x_{i}(0) \in[0,1] .
$$

These dynamical systems have recently been introduced in [8] and correspond to the best-response dynamics, i.e., the myopic response of agents for maximizing their own costs, given the actions of their neighbors, for a class of strategic games, c.f. Section IV. We will frequently refer to (2) as the piecewise linear best-response dynamical systems of strategic interactions. Note that according to (2), each agent aims at providing a unit of the resource and invests only if this unit is not provided, collectively by itself and its neighbors. The rate of contributions of each agent is inversely proportional to the investments of others.

Many scenarios that arise in economics (e.g. Cournot competition [8]) and engineering (e.g. private provision of public goods and investment in security [12]) can be formulated as such dynamical systems. As in other switched systems, in general, studying the convergence of these dynamical systems is challenging. For the scenarios where the influence of the neighbors is not substantial, the convergence of (2) has been established in [8]. Let us recall this result after introducing some notations as well as terminology.

Definition 3.1: (Active and passive sets): Let $\boldsymbol{x}=$ $\left(x_{1}, \ldots, x_{n}\right) \in[0,1]^{n}$. We say that agent $v_{i} \in V$ is active if $1-\sum_{j=1}^{n} a_{i j} x_{j}>0$ and passive otherwise. We denote by $\mathrm{S}_{\text {active }}, \mathrm{S}_{\text {passive }}:[0,1]^{n} \rightrightarrows V$, where $\mathrm{S}_{\text {active }}(\boldsymbol{x}) \subset V$ and $\mathrm{S}_{\text {passive }}(\boldsymbol{x}) \subset V$ are the subsets of active and passive agents, respectively. Given a set of active agents $V_{\mathrm{a}} \subset V$, we call the set

$$
X_{\text {active }}\left(V_{\mathrm{a}}\right)=\left\{\boldsymbol{x} \in[0,1]^{n} \mid \mathrm{S}_{\text {active }}(\boldsymbol{x})=V_{\mathrm{a}}\right\},
$$

the $V_{\mathrm{a}}$-set. The $V_{\mathrm{p}}$-set is defined similarly.

From Definition 3.1, it follows that $X_{\text {active }}\left(V_{\mathrm{a}}\right) \cup$ $X_{\text {passive }}\left(V \backslash V_{\mathrm{a}}\right)=[0,1]^{n}$. Given $V_{\mathrm{a}} \subset V$, one can relabel the rows and the columns of the adjacency matrix $A$ and partition it into active and passive blocks, $A=\left(\begin{array}{cc}A_{a} & A_{a p} \\ A_{p a} & A_{p}\end{array}\right)$, where the subindices $a$ and $p$ correspond to active and passive, respectively. Clearly, when following the evolutions of (2), each agent can switch from being active to being passive, and vice versa. In particular, it is not hard to see that when the influence of neighbors is large, these switches can happen quite frequently. The following result characterizes an interesting class where (2) is asymptotically stable [8].

Theorem 3.2: (Sufficient conditions for asymptotic stability of (2)): Suppose $\mathcal{G}$ is undirected. If $\lambda_{\min }(\mathrm{I}+A)>0$, then (2) has a unique equilibrium which is asymptotically stable.

When the conditions of Theorem 3.2 are violated, as characterized in [8], only a subset of the set of equilibria are stable. Hence, studying the stability properties of (2) for such scenarios becomes difficult, since these dynamical systems can become unstable on some active set $X_{\text {active }}\left(V_{\mathrm{a}}\right), V_{\mathrm{a}} \subset V$. Nevertheless, the trajectories may only visit these unstable regions for a finite time and still be convergent. In fact, when the underlying network is undirected, this is indeed the case and these dynamical systems are always convergent, regardless of the value of the weights on the adjacency matrix. We establish this result in the next section.

\section{ASYMPTOTIC CONVERGENCE OF MYOPIC STRATEGIC INTERACTION DYNAMICS}

We start this section by the construction of a game whose best-response dynamics is the dynamical system (2). Before stating the main result of this section, we recall the definition of a potential game [19]. Consider a game $G$ with players $\left\{v_{1}, \ldots, v_{n}\right\}$ with the strategy sets, respectively, $X_{1}, \ldots, X_{n}$ and payoff functions $u_{1}, \ldots, u_{n}$, where $u_{i}: X_{1} \times \ldots \times X_{n} \rightarrow$ $\mathbb{R}$, where $i \in\{1, \ldots, n\}$. We denote by $v_{-i}$ the set of all players, except $v_{i}$. Similarly, when $v_{i}$ chooses the strategy $x_{i}$, we denote by $x_{-i}$ the strategy of all other players. The game $G$ is called potential if there exists a function $P: X_{1} \times \ldots \times$ $X_{n} \rightarrow \mathbb{R}$ such that for every player $v_{i}$ and for every strategy $x_{i}, z_{i} \in X_{i}$ of $v_{i}$ and $x_{-i} \in X_{-i}$ of $v_{-i}$ we have

$$
u_{i}\left(x_{i}, x_{-i}\right)-u_{i}\left(z_{i}, x_{-i}\right)=P\left(x_{i}, x_{-i}\right)-P\left(z_{i}, x_{-i}\right),
$$


i.e., improvements for any player increases the function $P$.

Consider now a game $G_{\mathrm{ml}}$, defined over an undirected graph $\mathcal{G}$, with the set of players $V$, the strategy set of each player being $[0,1]$, and the payoff of the $i$ th player being $u_{i}:[0,1]^{n} \rightarrow \mathbb{R}$, where

$$
u_{i}\left(x_{i}, x_{-i}\right)=-\frac{1}{2} x_{i}^{2}-x_{i} \sum_{j \in \mathcal{N}_{i}} a_{i j} x_{j}+x_{i},
$$

which this player aims to maximize. Given the strategies of its neighbors, the best-response strategy $x_{i}^{*} \in[0,1]$ of the $i$ th player is then

$$
x_{i}^{*}= \begin{cases}0 & \sum_{j \in \mathcal{N}_{i}} a_{i j} x_{j} \geq 1, \\ 1-\sum_{j \in \mathcal{N}_{i}} a_{i j} x_{j} & \text { otherwise }\end{cases}
$$

and hence the dynamical system (2) is precisely the bestresponse dynamical system for $G_{\mathrm{ml}}$. Note that the construction of $G_{\mathrm{ml}}$ is certainly not unique (see [8] for more examples). Our first result shows that choosing $G_{\mathrm{ml}}$ as above has its advantages.

Proposition 4.1: $\left(G_{\mathrm{ml}}\right.$ is a potential game): The game $G_{\mathrm{ml}}$, defined over an undirected graph, with payoff functions given by (3), is a potential game with the potential function $P$ : $[0,1]^{n} \rightarrow \mathbb{R}$ given by

$$
P\left(x_{1}, \ldots, x_{n}\right)=\sum_{i=1}^{n} \frac{1}{2}\left(-x_{i}^{2}-x_{i} \sum_{j \in \mathcal{N}_{i}} a_{i j} x_{j}+2 x_{i}\right) .
$$

The proof follows immediately from the definition of potential function above. One can also see that the game $G_{m l}$ with payoff functions (3) is strategically equivalent to a game where all players have the same (common) cost function (4), that is a team; following the construction that was first introduced in [26], simply add to (3) for $i$-th player $x_{j}$ 's for $j \neq i$ so that the resulting (modified) $u_{i}$ 's are identical for all $i$ (which would be $P$ ). The construction of $G_{\mathrm{ml}}$ has another useful property that allows us to conclude that (2) is indeed convergent.

Theorem 4.2: (Asymptotic convergence to the set of equilibria): The trajectories of (2) are globally asymptotically convergent to the set of equilibria of $G_{\mathrm{ml}}$.

Proof: Since $\dot{x}_{i}(t) \leq 1-x_{i}(t)$ for all $t \in \mathbb{R}_{\geq 0}$, we have that $x_{i}(t) \leq 1$. Similarly, since $\dot{x}_{i}(t) \geq-x_{i}(t)$, we also have that $x_{i}(t) \geq 0 t \in \mathbb{R}_{\geq 0}$. As a result, the trajectories of (2) are bounded. Moreover, the potential function (4) is component-wise strictly concave. Thus, by invoking the result of [24, Page 347], $P$ serves as a Lyapunov function and the best-response dynamical system (2) is convergent.

Figures 1 demonstrates this result for a cycle network with 10 agents. By providing an independent proof, we demonstrate in the next example that the result of Theorem 4.2 is highly nontrivial, even for small size networks.

Example 4.3: (Stability outside the sufficient conditions of Theorem 3.2): Consider the best-response dynamical system on the path graph with three nodes, given by

$$
\begin{aligned}
& \dot{x}_{j}= \begin{cases}1-x_{j}-\delta x_{2} & 1-\delta x_{2}>0, \\
-x_{j} & \text { otherwise; }\end{cases} \\
& \dot{x}_{2}= \begin{cases}1-x_{2}-\delta\left(x_{1}+x_{3}\right) & 1-\delta\left(x_{1}+x_{3}\right)>0, \\
-x_{2} & \text { otherwise; }\end{cases}
\end{aligned}
$$

where $\delta \in[0,1]$. We denote by $A$ the adjacency matrix associated to this graph.

Note that, by Theorem 4.2, (5) is convergent to its set of equilibria. Let us show this by a direct proof. Since $\lambda_{\min }(\mathrm{I}+$ $\delta A)=1-\sqrt{2} \delta$, the result of Theorem 3.2 leads to asymptotic stability of (5) when $0 \leq \delta<\frac{1}{\sqrt{2}}$. We next show that more is true and (5) is in fact asymptotically stable for also any $\frac{1}{\sqrt{2}} \leq \delta<1$. Toward that end, we first prove that the subspace

$$
W=\left\{\boldsymbol{x}=\left(x_{1}, x_{2}, x_{3}\right) \in[0,1]^{3} \mid x_{1}+x_{3} \geq \frac{1}{\delta} \text { and } x_{2} \leq \frac{1}{2 \delta}\right\},
$$

is invariant under the flow. Note that since $x_{1}+x_{3} \geq 1$, agent 2 is passive and thus when $\boldsymbol{x}(0) \in W$, for all $t \in \mathbb{R}_{>0}$, $x_{2}(t) \leq x_{2}(0)$. Moreover, since $x_{2} \leq \frac{1}{2 \delta}$ and in $W$

$$
\dot{x}_{1}+\dot{x}_{3}=2-\left(x_{1}+x_{3}\right)-2 \delta x_{2},
$$

we conclude that $x_{1}(t)+x_{3}(t) \leq x_{1}(0)+x_{3}(0)$, for all $t \in \mathbb{R}_{>0}$, thus proving our claim. Next, we show that any trajectory starting from any initial condition $\boldsymbol{x} \notin W$, will eventually enter $W$. Note that (5) has no equilibrium in the region $U=\left\{\boldsymbol{x}=\left(x_{1}, x_{2}, x_{3}\right) \in[0,1]^{3} \mid x_{1}+x_{3}<\frac{1}{\delta}\right\}$; moreover, $\mathrm{I}_{3}+\delta A$ has one negative eigenvalue and thus any trajectory starting from an initial condition $\boldsymbol{x}(0) \in U$ will eventually leave this region, entering $W$. Also, since agent 2 is passive in $[0,1]^{3} \backslash(U \cup W)$, any trajectory starting from this region will eventually leave this region, yielding the result. Next, since the flow is invariant in $W$, using a Lyapunov argument on this region, we conclude that any flow entering $W$ will asymptotically converge.

An interesting by-product of our treatment is a proof of stability for some challenging classes of piecewise dynamical systems, as we state in the following remark.

Remark 4.4: (Stability analysis of a class of On-Off Systems): We introduce a class of decentralized On-Off Systems (OFSs), motivated by the single-input version studied in [17, Section VI]. Consider the control system

$$
\left\{\begin{array}{l}
\dot{\boldsymbol{x}}(t)=\boldsymbol{x}(t)+u(t) \\
\boldsymbol{y}(t)=C \boldsymbol{x}(t)
\end{array}\right.
$$

where $\boldsymbol{x}(t) \in \mathbb{R}^{n}, n \in \mathbb{Z}_{\geq 1}, C \in \mathbb{R}^{n \times n}$, and $u(t) \in \mathbb{R}^{n}$ is defined via $u_{i}(t)=\max \left\{0, y_{i}(t)-d_{i}\right\}, i \in\{1, \ldots, n\}$, $d_{i} \in \mathbb{R}_{>0}$. Additionally, assume that $C$ is the adjacency matrix associated with an undirected connected graph $\mathcal{G}$ with $\sum_{j=1}^{n} c_{i j}=1$, for all $i \in\{1, \ldots, n\}$. The control input is thus decentralized over $\mathcal{G}$. Next, consider a potential game with $n$ players, where the $i$ th player's strategy set is $0 \leq x_{i} \leq d_{i}$ and this player is maximizing the function $u_{i}\left(x_{i}, x_{-i}\right)=$ $-\frac{1}{2} x_{i}^{2}-x_{i} \sum_{j \in \mathcal{N}_{i}} c_{i j} x_{j}+d_{i} x_{i}$. One can easily observe that the control system (6) corresponds to the best-response dynamical system for this game; furthermore, similar to the construction in Proposition 4.1, the corresponding potential function is indeed component-wise concave. Hence, (6) is asymptotically convergent to its set of equilibria. Note that proving this result directly appears to be nontrivial.

Before concluding this section, we collect some interesting features of the set of equilibria of (2). 


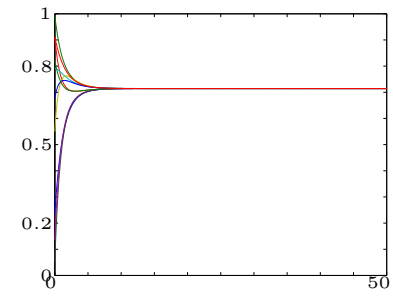

(a)

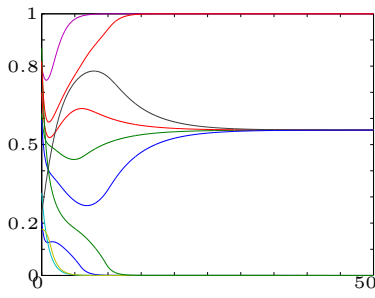

(b)

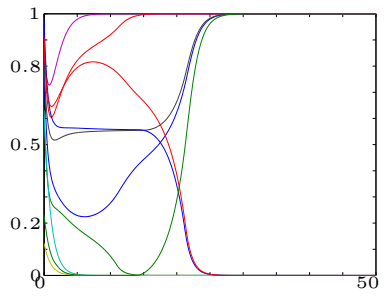

(c)

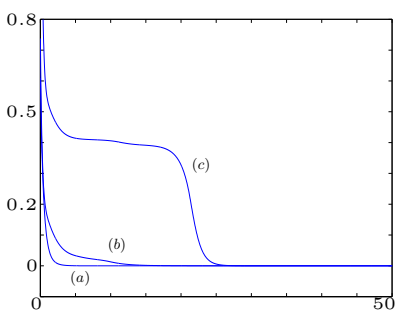

(d)

Fig. 1. (a) and (b) show the evolutions of (2) for a cycle graph consisting of 10 nodes. In (a), the weights on each edge is 0.2 and the initial condition is $x_{0}=(0.2363,0.9943,0.9093,0.7908,0.1351,0.5503,0.0538,0.6766,0.8223,0.9114)$; the evolutions are globally asymptotically convergent to the unique equilibrium $x^{*}=0.71431_{10}$, as per Theorem 3.2, and also Theorem 4.2. In (b) and (c), the weights on each edge is 0.8 and the initial conditions are, respectively, $x_{0}=(0.2049,0.6188,0.6992,0.3148,0.8048,0.1780,0.2413,0.5947,0.8682,0.8187)$ and $x_{0}=(0.7863,0.6014,0.9470,0.6312,0.8788,0.1227,0.8135,0.9776,0.2312,0.9127) ;$ the evolutions, respectively, asymptotically converge to $x^{*}=$ $(0,0.5556,0.5556,0,1,0,0.5556,0.5556,0,1)$ and $x^{*}=(1,0,1,0,1,0,1,0,1,0)$, as predicted by Theorem 4.2 . As the plots show, the behavior of the agents, in terms of being active or passive, is complex. Finally, (d) shows the evolution of the Lyapunov function in absolute value, constructed via (4), for each case.

\section{A. Unique equilibrium in directed networks}

We first provide a result which shows that a subclass of the dynamics (2) on directed graphs is convergent to a unique equilibrium. Note that since the graph is directed, Theorem 4.2 cannot be used to conclude convergence, as the potential game structure is lost.

Proposition 4.5: (Sufficient conditions for convergence to unique equilibrium for the directed network): Let $\mathcal{G}$ be any weighted directed graph with the set of vertices $V$. If $\max _{v_{i} \in V}\left(d_{\mathrm{out}}^{\mathrm{w}}\left(v_{i}\right)\right)<1$, the Nash equilibrium of the strategic network game is unique and (2) is convergent to it.

Proof: Recall that the trajectories of (2) are bounded. Let $n=|V|$. By assumption, $1-\sum_{j=1}^{n} a_{i j} x_{j}>0$, for all $i \in$ $\{1, \ldots, n\}$ and $x_{j} \in[0,1]$. Thus the best-response dynamical system (2) reads

$$
\dot{\boldsymbol{x}}=\mathbf{1}-A \boldsymbol{x}-\boldsymbol{x},
$$

where $\boldsymbol{x}=\left(x_{1}, \ldots, x_{n}\right)^{T}$ and $A$ is the corresponding weighted adjacency matrix. Any equilibrium of this linear system thus satisfies

$$
\left(A+\mathrm{I}_{n}\right) \boldsymbol{x}=\mathbf{1} .
$$

By Gersgorin disks Theorem [27, Theorem 6.1.1], and since $\mathcal{G}$ has no self-loops and $A \in \mathbb{R}_{\geq 0}^{n \times n}$,

$$
\begin{aligned}
\operatorname{spec}\left(A+\mathrm{I}_{n}\right) & \subset \bigcup_{i \in\{1, \ldots, n\}}\left\{z \in \mathbb{C} \mid\|z-1\|_{\mathbb{C}} \leq \sum_{j=1}^{n} a_{i j}\right\} \\
& \subset\left\{z \in \mathbb{C} \mid\|z-1\|_{\mathbb{C}}<1\right\},
\end{aligned}
$$

where the last inclusion holds by the assumption made. Thus $A+\mathrm{I}_{n}$ is invertible and the solution to (7) is indeed unique. The asymptotic convergence of the best-response dynamics also follows immediately from the fact that the eigenvalues of this matrix all have positive real parts.

\section{B. Abundance of equilibria}

We next show that the dynamical system (2) can have a continuum of equilibria. Consider an undirected path graph with $n \in \mathbb{Z}_{\geq 1}$ vertices, where every edge is weighted by $\delta \in[0,1]$. Note that for any choice of $\delta$, the matrix I $+\delta A$, where $A$ is the adjacency matrix, has at most one zero eigenvalue. This is because the eigenvalues of $A$ can be written in terms of Chebyshev polynomials [28], which are orthogonal polynomials and thus have distinct eigenvalues. Let us now consider the case where $n=4$. The set of eigenvalues of $A$ is given by

$$
\{-1.6180,-0.6180,0.6180,1.6180\} \text {. }
$$

Let us now choose $\delta=-1 / \lambda_{\min }(A) \cong 0.6180$. Then any point in the set $\left\{x \in[0,1]^{4} \mid(\mathbf{I}+\delta A) \boldsymbol{x}=\mathbf{1}\right\}$ is an equilibrium of (2).

\section{Emergence of unstable equilibria in stable submanifolds}

Consider a path graph with $n=4$ and let $\delta=0.9$, which is larger than the threshold for which Theorem 3.2 applies. Using the Stable Manifold Theorem [29], the stable submanifold $S_{\text {stable }}$ is of dimension 3. Let us take the initial condition to be $x_{0}=(0.3717,0.6015,0.6015,0.3717)^{T} \in$ $S_{\text {stable. Figure 2(a) shows the trajectories of (2) starting }}$ from $x_{0}$. These trajectories are asymptotically convergent to $x^{*}=(0.9174,0.0917,0.0917,0.9174)^{T} \in S_{\text {stable }}$. Nevertheless, with small perturbations in the initial conditions, orthogonal to $S_{\text {stable }}$, the trajectories leave $S_{\text {stable }}$; see Figure 2(b).

\section{Social Relation and the Behavior of the}

\section{DYNAMICS}

In previous sections, we have focused mainly on stability analysis of the dynamical system (2) for the case where the underlying graph is undirected. In this section, we further explore the asymptotic behaviors of those dynamical systems by considering the directed case. In the upcoming discussion, unless otherwise stated, we assume that the underlying graph $\mathcal{G}=(V, E)$ is a directed weighted graph. Let us define the two concepts of social dominance and social equivalence.

Definition 5.1: (Social equivalence and social dominance): We say that agents $v_{i} \in V$ and $v_{j} \in V$ are socially equivalent if $a_{i \ell}=a_{j \ell}$ for all $v_{\ell} \in V$. We say that $v_{i}$ is socially dominated by $v_{j}$ if $a_{i \ell} \leq a_{j \ell}$ for all $v_{\ell} \in V$.

Note that in the case of equally weighted graphs, $v_{i}$ and $v_{j}$ are socially equivalent and $v_{i}$ is socially dominated by $v_{j}$ are equivalent to $\mathcal{N}_{i}^{+}=\mathcal{N}_{j}^{+}$and $\mathcal{N}_{i}^{+} \subseteq \mathcal{N}_{j}^{+}$, respectively.

Social equivalency and social dominance have interesting implications. The first observation is that social equivalence implies consensus. 


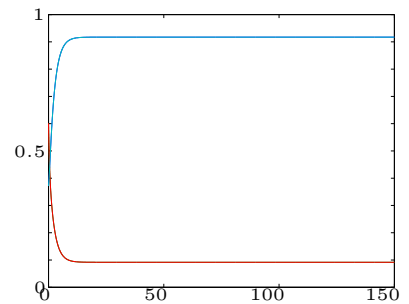

(a)

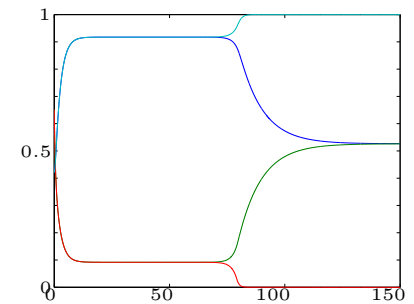

(b)

Fig. 2. (a) and (b) show the trajectories of (2) for a path graph with 4 agents with the initial conditions of $x_{0}$ and $x_{0}+0.051_{4}$.

Proposition 5.2: (Social equivalence implies consensus): Consider the best-response dynamics on a digraph $\mathcal{G}=$ $(V, E, A)$. If $v_{i}, v_{j}$ are socially equivalent, $\lim _{t \rightarrow \infty}\left(x_{i}(t)-\right.$ $\left.x_{j}(t)\right)=0$ for any initial condition $\boldsymbol{x}(0) \in \mathbb{R}^{n}$.

Proof: Note that if $v_{i}$ and $v_{j}$ are socially equivalent, then from (2), it follows that $\dot{x}_{i}-\dot{x}_{j}=-\left(x_{i}-x_{j}\right)$.

Our second result is related to the implications of social dominance. We first show that if one agent is socially dominated by another agent, and at some point of time the dominated agent $v_{i}$ invests more (i.e. $x_{i}(t) \geq x_{j}(t)$ ), then it should invest more than the other agent indefinitely. Subsequently, we show that if this does not happen, i.e. a socially dominated $v_{i}$ keeps working less than $v_{j}$ forever, then either the neighbors of $v_{i}$ are hard-working or the neighbors of $v_{j}$ (other than $v_{i}$ ) are asymptotically lazy, in the sense of the size of their contributions.

Proposition 5.3: (Implications of social dominance): Consider a digraph $\mathcal{G}=(V, E, A)$ and the associated dynamics (2), and suppose that $v_{i} \in V$ is dominated by $v_{j} \in V$.

a. If $x_{i}(T) \geq x_{j}(T)$ for some time $T \geq 0$, then $x_{i}(t) \geq x_{j}(t)$ for all $t \geq T$.

b. Suppose that the weights on $A$ are uniform over the edges, i.e. for any $a_{i \ell}>0$, we have $a_{i \ell}=\delta$ for some $\delta>0$. If for all $t \geq 0$, we have $x_{i}(t)<x_{j}(t)$, then

$$
\liminf _{t \rightarrow \infty}\left(\sum_{\ell \in \mathcal{N}_{j}^{+} \backslash \mathcal{N}_{i}^{+}} x_{\ell}(t)\right)\left(1-\delta \sum_{\ell \in \mathcal{N}_{i}^{+}} x_{\ell}(t)\right) \leq 0
$$

Proof: Let us start with the proof of the first statement.

a) Since $v_{i}$ is socially dominated by $v_{j}$, we have

$$
\begin{aligned}
& \dot{x}_{j}(t)=f_{j}(\boldsymbol{x})=\max \left(1-\sum_{v_{\ell} \in V} a_{j \ell} x_{\ell}(t), 0\right)-x_{j}(t), \\
& \dot{x}_{i}(t)=f_{i}(\boldsymbol{x})=\max \left(1-\sum_{v_{\ell} \in V} a_{i \ell} x_{\ell}(t), 0\right)-x_{i}(t) .
\end{aligned}
$$

Note that $f_{i}$ and $f_{j}$ are continuous functions on $[0,1]^{n}$. Also, since $v_{i}$ is socially dominated by $v_{j}$, for any point in $\boldsymbol{x} \in[0,1]^{n}$, we have that $f_{i}(\boldsymbol{x}) \geq f_{j}(\boldsymbol{x})$. Thus, if for some time instance $T \geq 0$, we have $x_{i}(T) \geq x_{j}(T)$, then by the comparison principle (see [25, Lemma 3.4]), it follows that $x_{i}(t) \geq x_{j}(t)$ for all $t \geq T$. b) We next prove the second statement. By (8), we have

$$
\begin{aligned}
\dot{x}_{i}(t)-\dot{x}_{j}(t)= & \left(\max \left(1-\delta \sum_{\ell \in \mathcal{N}_{i}^{+}} x_{\ell}(t), 0\right)\right. \\
& -\max \left(1-\delta \sum_{\ell \in \mathcal{N}+_{j}} x_{\ell}(t), 0\right) \\
& +\left(x_{j}(t)-x_{i}(t)\right) .
\end{aligned}
$$

Thus, by integrating both sides of the above equation,

$$
\begin{aligned}
& \left(x_{i}(t)-x_{j}(t)\right)-\left(x_{i}(0)-x_{j}(0)\right) \\
= & \int_{0}^{t} \max \left(1-\delta \sum_{\ell \in \mathcal{N}_{i}^{+}} x_{\ell}(s), 0\right) d s \\
- & \int_{0}^{t} \max \left(1-\delta \sum_{\ell \in \mathcal{N}_{j}^{+}} x_{\ell}(s), 0\right) d s+\int_{0}^{t}\left(x_{j}(s)-x_{i}(s)\right) d s
\end{aligned}
$$

Thus

$$
\begin{aligned}
& \left(x_{i}(t)-x_{j}(t)\right)-\left(x_{i}(0)-x_{j}(0)\right) \\
& \geq \int_{0}^{t} \max \left(1-\delta \sum_{\ell \in \mathcal{N}_{i}^{+}} x_{\ell}(s), 0\right) d s \\
& \quad-\int_{0}^{t} \max \left(1-\delta \sum_{\ell \in \mathcal{N}_{j}^{+}} x_{\ell}(s), 0\right) d s=\int_{0}^{t} g(s) d s,
\end{aligned}
$$

where $g(s)=\max \left(1-\delta \sum_{\ell \in \mathcal{N}_{i}^{+}} x_{\ell}(s), 0\right)-\max (1-$ $\left.\delta \sum_{\ell \in \mathcal{N}_{j}^{+}} x_{\ell}(s), 0\right)$ and the inequality follows from the fact that $x_{j}(s)>x_{i}(s)$ for all $s \geq 0$. Since $x_{i}(t)-$ $x_{j}(t)$ is bounded, it follows that $\int_{0}^{\infty} g(s) d s<\infty$. Also, note that $g(s)$ is a uniformly continuous function as $x_{\ell}(s)$ is differentiable for all $\ell$ and the derivatives are bounded (by \pm 1 ). Thus, by Barbalat's lemma ([25], Lemma 8.2) it follows that $\lim _{t \rightarrow \infty} g(s)=0$. Now the claim is that $\lim _{t \rightarrow \infty} g(s)=0$ implies that $\liminf \operatorname{in}_{t \rightarrow \infty}\left(\sum_{\ell \in \mathcal{N}_{j}^{+} \backslash \mathcal{N}_{i}^{+}} x_{\ell}(s)\right)\left(1-\delta_{\ell \in \mathcal{N}_{i}^{+}}\right) \leq 0$. For this, first note that if $\max \left(1-\delta \sum_{\ell \in \mathcal{N}_{j}^{+}} x_{\ell}(s), 0\right)>0$, then

$$
\begin{aligned}
g(s) & =\delta \sum_{\ell \in \mathcal{N}_{j}^{+} \backslash \mathcal{N}_{i}^{+}} x_{\ell}(s) \\
& \geq \delta\left(\sum_{\ell \in \mathcal{N}_{j}^{+} \backslash \mathcal{N}_{i}^{+}} x_{\ell}(s)\right)\left(1-\delta \sum_{\ell \in \mathcal{N}_{i}^{+}} x_{\ell}(s)\right),
\end{aligned}
$$


and if $\max \left(1-\delta \sum_{\ell \in \mathcal{N}_{j}^{+}} x_{\ell}(s), 0\right)=0$, it follows that

$$
\begin{aligned}
g(s) & \geq\left(1-\delta \sum_{\ell \in \mathcal{N}_{i}^{+}} x_{\ell}(s)\right) \\
& \geq\left(\sum_{\ell \in \mathcal{N}_{j}^{+} \backslash \mathcal{N}_{i}^{+}} x_{\ell}(s)\right)\left(1-\delta \sum_{\ell \in \mathcal{N}_{i}^{+}} x_{\ell}(s)\right) .
\end{aligned}
$$

The above results shed more lights on the asymptotic behavior of the dynamics (2). As an example of such implications, let $\mathcal{G}=(V, E)$ be a complete $q$-partite graph, i.e. an undirected graph $\mathcal{G}=(V, E)$ with $V$ being disjoint union of $q$ nonempty sets $V_{1}, V_{2}, \ldots, V_{q}$ (which are commonly referred to as components) with $E=\bigcup_{\ell_{1} \neq \ell_{2}} V_{\ell_{1}} \times V_{\ell_{2}}$. Note that any two vertices belonging to the same component have the same set of neighbors. Therefore, by Proposition 5.2, we have $\lim _{t \rightarrow \infty} x_{i}(t)=\lim _{t \rightarrow \infty} x_{j}(t)$ for any $i, j \in V_{\ell}$ for some $\ell \in\{1, \ldots, q\}$. As a result, if the sizes of the components are different, one may expect that for a random initial condition the asymptotic value of the dynamics (2) have $q$ distinct values. Note that $q$ is the chromatic number of a complete $q$-partite graph. We conjecture that this holds true for a random graph $\mathcal{G}$, i.e. the distinct values of the asymptotic value of dynamics (2) started at a random initial condition is approximately equal to the chromatic number of $\mathcal{G}$.

\section{CONCLUSiOnS AND FUTURE WORK}

We have shown that the dynamical systems of piecewise linear strategic interaction dynamics on networks are convergent on any undirected graph topology. Our proof relies on casting such dynamical systems as the best-response dynamics of a class of potential games with component-wise concave potential functions. We have also introduced the novel notions of social equivalence and social dominance and have characterized and studied their implications on the asymptotic behavior of dynamical systems. Many avenues of future work appear to be open, including the study of asymmetric interactions (directed case), the design of other classes of strategic games which are not completely myopic, further investigation on the casting of the strategic interaction dynamics as a distributed strategy for approximating the chromatic number, and studying scenarios with time-varying networks and their applications to stability analysis of switched dynamical systems.

\section{ACKNOWLEDGMENTS}

The authors are thankful to Professors Daniel Liberzon and Cedric Longbort for insightful discussions. The work of the first author was partially supported by the Natural Sciences and Engineering Research Council of Canada. The work of Gharesifard and Başar was supported in part by the NSA through the Information Trust Institute of the University of Illinois, and in part by the AFOSR MURI Grant FA9550-101-0573. The work of Touri and Shamma was supported by AFOSR/MURI FA9550-09-1-0538.

\section{REFERENCES}

[1] B. Gharesifard, B. Touri, T. Başar, and C. Langbort, "Distributed optimization by myopic strategic interactions and the price of heterogeneity," in Proc. IEEE Conf. on Decision and Control, (Florence, Italy), pp. 1174-1179, 2013.
[2] S. H. Strogatz, "From Kuramoto to Crawford: Exploring the onset of synchronization in populations of coupled oscillators," PhysicaD, vol. 143 , no. 1, pp. 1-20, 2000.

[3] F. Bullo, J. Cortés, and S. Martínez, Distributed Control of Robotic Networks. Applied Mathematics Series, Princeton University Press, 2009. Electronically available at http://coordinationbook.info.

[4] M. O. Jackson, Social and Economic Networks. Princeton, NJ: Princeton University Press, 2008.

[5] N. Li and J. R. Marden, "Designing games for distributed optimization," in Proc. IEEE Conf. on Decision and Control, (Orlando, FL), pp. 2434 2440, Dec. 2011.

[6] F. Kelly, A. Maulloo, and D. Tan, "Rate control for communication networks: Shadow prices, proportional fairness and stability," Journal of Operations Research, vol. 49, no. 3, pp. 237-252, 1998.

[7] S. Goyal and M. Kearns, "Competitive contagion in networks," in Proceedings of the 44th Symposium on Theory of Computing, pp. 759774, ACM Press, 2012.

[8] Y. Bramoullé, R. Kranton, and M. D'Amours, "Strategic interaction and networks," American Economic Review, vol. 104, no. 3, pp. 898-930, 2014.

[9] M. Granovetter, "Threshold models of collective behavior," American Journal of Sociology, vol. 83, no. 6, pp. 1420-1443, 1978.

[10] R. Cooper and A. John, "Coordinating coordination failures in Keynesian models," The Quarterly Journal of Economics, vol. 103, no. 3, pp. $441-463,1988$.

[11] J. Kleinberg, "Cascading behavior in social and economic networks," in Proceedings of the Fourteenth ACM Conference on Electronic Commerce, (New York, NY), pp. 1-4, 2013.

[12] Y. Bramoullé and R. Kranton, "Public goods in networks," Journal of Economic Theory, vol. 135, no. 1, pp. 478-494, 2007.

[13] C. Ballester, A. Calvó-Armengol, and Y. Zenou, "Who's who in networks. Wanted: the key player," Econometica, vol. 74, no. 5, pp. 14031417, 2006.

[14] G. Angeletos and A. Pavan, "Transparency of information and coordination in economies with investment complementarities," American Economic Review Papers and Proceedings, vol. 94, no. 2, pp. 91-98, 2004.

[15] M. Belhaj and F. Deroïan, "Strategic interaction and aggregate incentives," Journal of Mathematical Economics, vol. 49, no. 3, pp. 183-188, 2013.

[16] M. di Bernardo, C. Budd, A. Champneys, and P. Kowalczyk, PiecewiseSmooth Dynamical Systems. New York: Springer, 2008.

[17] J. M. Goncalves, A. Megretski, and M. A. Dahleh, "Global analysis of piecewise linear systems using impact maps and surface Lyapunov functions," IEEE Transactions on Automatic Control, vol. 48, no. 12, pp. 2089-2106, 2003.

[18] M. Johansson and A. Rantzer, "Computation of piecewise quadratic Lyapunov functions for hybrid systems," IEEE Transactions on Automatic Control, vol. 43, no. 4, pp. 555-559, 1998.

[19] D. Monderer and L. S. Shapley, "Potential games," Games and Economic Behavior, vol. 14, pp. 124-143, 1996.

[20] M. Hirsch, "Systems of differential equations which are competitive or cooperative: I. Limit sets," SIAM Journal on Mathematical Analysis, vol. 13 , no. 2, pp. 167-179, 1982.

[21] M. Hirsch, "Systems of differential equations that are competitive or cooperative: II. Convergence almost everywhere," SIAM Journal on Mathematical Analysis, vol. 16, no. 3, pp. 423-439, 1985.

[22] T. Başar and G. J. Olsder, Dynamic Noncooperative Game Theory. SIAM, 2 ed., 1999.

[23] H. P. Young, Strategic Learning and its Limits. Oxford, UK: Oxford University Press, 2004.

[24] M. Benaïm, J. Hofbauer, and S. Sorin, "Stochastic approximation and differential inclusions," SIAM Journal on Control and Optimization, vol. 44, no. 1, pp. 328-348, 2006.

[25] H. K. Khalil, Nonlinear Systems. Prentice Hall, 2 ed., 1995.

[26] T. Başar and Y. C. Ho, "Informational properties of the Nash solutions of two stochastic nonzero-sum games," Journal of Economic Theory, vol. 7, no. 4, pp. 370-387, 1974.

[27] R. A. Horn and C. R. Johnson, Matrix Analysis. Cambridge University Press, 1985.

[28] N. Biggs, Algebraic Graph Theory. Cambridge University Press, 2 ed., 1994.

[29] V. Arnol'd, Mathematical Methods of Classical Mechanics. No. 60 in Graduate Texts in Mathematics, Springer, 2 ed., 1989. 